\title{
Development of real time internet of things (IoT) based air quality monitoring system
}

\author{
Huzein Fahmi Hawari, Aideed Ahmad Zainal, Mohammad Radzi Ahmad \\ Department of Electrical and Electronic Engineering, Universiti Teknologi PETRONAS, Malaysia
}

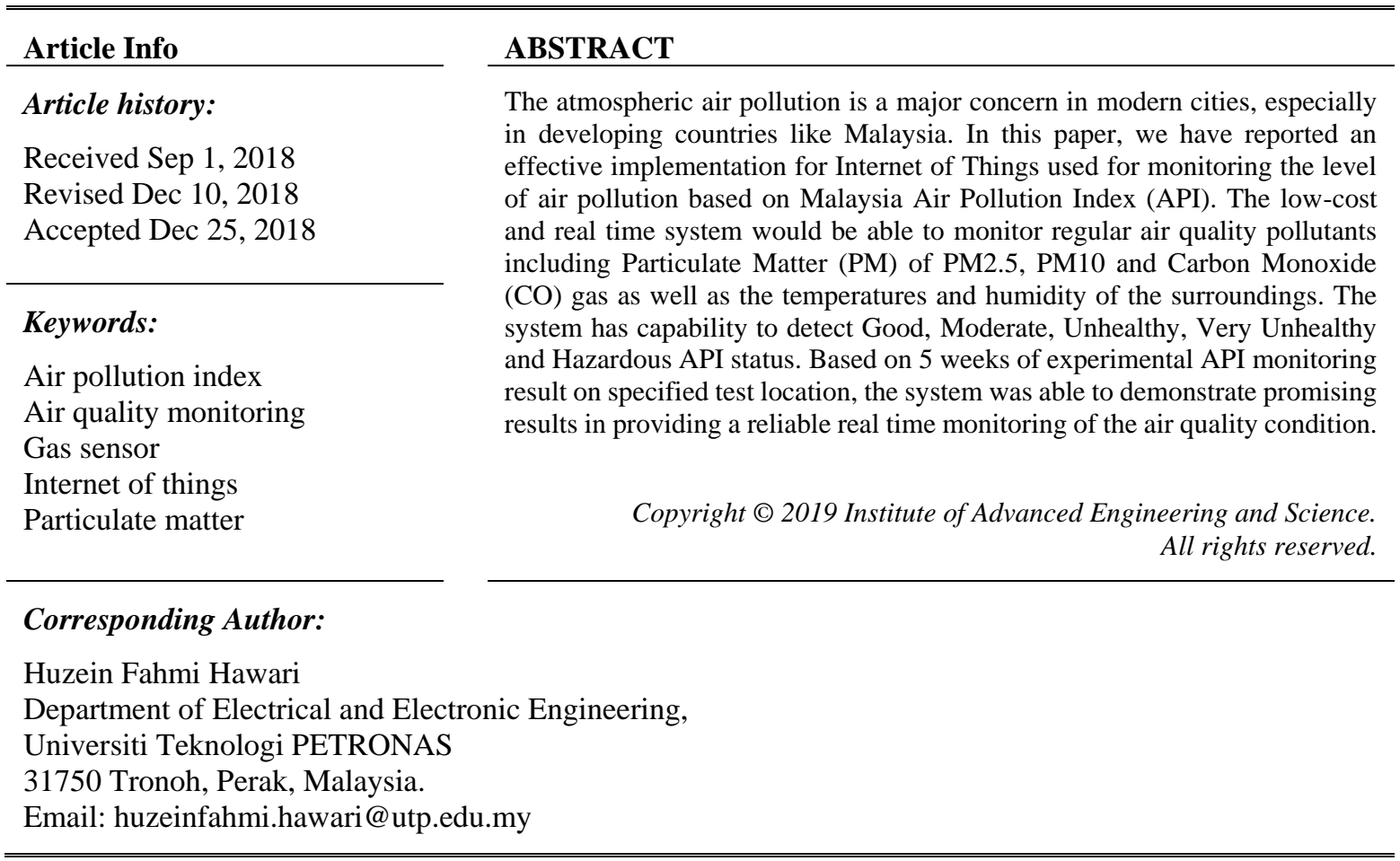

\section{INTRODUCTION}

Malaysia is one of the countries that have been severely affected by haze for the past 10 years. The number of acute respiratory disease cases is spiking approximately $60 \%$ because of haze [1]. Social awareness towards the surrounding air quality has increased the demand for real time air quality information. According to The Star on September $16^{\text {th }}, 2015$, Malaysians complaint on very short notice of school closing due to unhealthy API level which mainly caused by haze [1]. Current API system still using the same as the one established in 1993. Based on Malaysia Department of Environment, the data processed based on averaging time of 24 hours [2]. Hence, the current method is not accurate to represents the actual environment situation. A real time monitoring necessarily required in order carrying out immediate actions in case of unhealthy API level reached. Therefore, through this research, an air quality monitoring system has been developed using Arduino Yun Mini and ThingSpeak as the Internet of Things (IoT) platform in order to create a medium of sharing information for pollution monitoring; where the data management and control can be done conveniently through the internet with secured environment.

In addition, current stationary API monitoring system situated only at certain area gives less reliable results for the people who live distantly [3]. A portable concept of API monitoring will surely help to overcome this problem. Malaysia API monitoring system is incapable of measure PM2.5 and expected to be implemented in the system on 2020 while neighbor countries like Singapore already capable [3], [4]. Thus, PM2.5 sensor integrated in this system has an advantage on giving reliable functionality on common pollutants detection. A low-cost concept implemented in this system makes it affordable towards wide range of people. As per Table 1, usually, the stationary type of Air Quality Monitoring system consisting of Analytical Instruments is very expensive [5], [6]. 
Table 1. Comparison between analytical instruments and pollutants sensors [6], [7]

\begin{tabular}{ccc}
\hline Characteristics & Analytical instruments & Air quality sensors \\
\hline Cost & Very high & Fair \\
Size & Bulky & Compact \\
Resolution & Excellent & Reliable \\
Mobility & Difficult & Easy \\
\hline
\end{tabular}

A low-cost air quality monitoring system has been developed which only focused on dust pollutants monitoring by using Sharp GP2Y1010AU0F sensor [8]. A similar approach done with addition of Global System for Mobile Communications (GSM) modem integrated in the system [9]. However, the system monitoring was limited to PM10 pollutants only. In the other hand, a CO Gas Detector was designed based on ATMEGA8535 microcontroller, TGS 2600 sensor and Global System for Mobile (GSM) module [10]. Another project which focused on monitoring the $\mathrm{CO}$ concentration level with main aim on industrial purpose developed [11]. The system used PIC18F4550 microcontroller and integrated with MQ-7 CO sensor. The system consists of 3 parts of circuits which are power supply unit, signal conditioning unit, alarm, display and microcontroller unit.

An indoor air quality monitoring system developed with different approach which classified the pollutants as Volatile Organic Components (VOC) [12]. The system using mbed LPC1768 microcontroller based on 32-bit ARM cortex M3 processor integrated with the TGS 2602. The pollutants monitored which has been classified as VOC are Hydrogen, Ammonia, Ethanol, Hydrogen Sulfide and Toluene. Another sensor called Sense Air S8 used in the system mainly to measures Carbon Dioxide. Further, DHT22 used to monitor the temperature and humidity conditions.

Inspired by the Internet of Things trend, a device called 'Polluino' was developed to monitor the air quality [13]. It is enhanced with cloud-base management for increase the efficiency. Neglecting the low-cost aspect, it is equipped with 12 different types of gas sensors and 5 environmental sensors. Arduino microcontroller preferred to be used with its open source flexibility and integrated with Wi-Fi module, ESP8266, as its network communication medium.

AirSense, an Air Quality Monitoring Device (AQMD) which focus on low power and low-cost system implementation developed [14]. The system used Bluetooth HC-05 module to transmit data to smart phone device and share the data through the cloud. By using the MQ-135 gas sensor, the air monitored and the data uploaded to the cloud through ThingSpeak web service. Still, the connectivity between the smart phone and the AQMD will be limited up to a certain range as it using the Bluetooth communication medium.

In this project, combination of MQ-7 CO sensor, DHT22 temperature/humidity sensor, PM10 and PM2 sensor will be intregated in order to provide more detail environmental parameter compared to previous research. A GSM module is also intergrated in the system to carry out the real time monitoring function of distant notifications by Short Message Services (SMS). Whenever the measured values exceed the limits, SMS will be sent to the specific phone number to alert the user of the possible environmental health effect.

\section{RESEARCH METHOD}

The system designed to be able to measure several common air quality index and standardized the results with the Malaysia Air Pollutant Index to indicate the severity of the air quality. Hence, the selection of the hardware to be used in the system has been done to make sure that the hardware used is able to give results which can be standardized with the API. The air quality monitoring system compacted with 4 sensors integrated into it and each has their own functionality to measure $\mathrm{CO}$ pollutant, temperature, humidity, $\mathrm{PM}_{10}$ and $\mathrm{PM}_{2.5}$.

To ensure the reliability of the system, each of the sensors ensured to be calibrated. However, the only sensor required to be calibrated before fully integrated into the system is $\mathrm{CO}$ sensor while the others has been readily calibrated by the manufacturer. To make the air flow nearby the sensors regulated, a DC brushless fan installed in the device. This to make sure that the results obtained to be more valid. Details of the block system diagram of API monitoring is illustrated in Figure 1.

A structure of process flow has been designed and followed during the implementation of the system. The process flow planned thoroughly to ensure the research covering the whole objective of the project. 


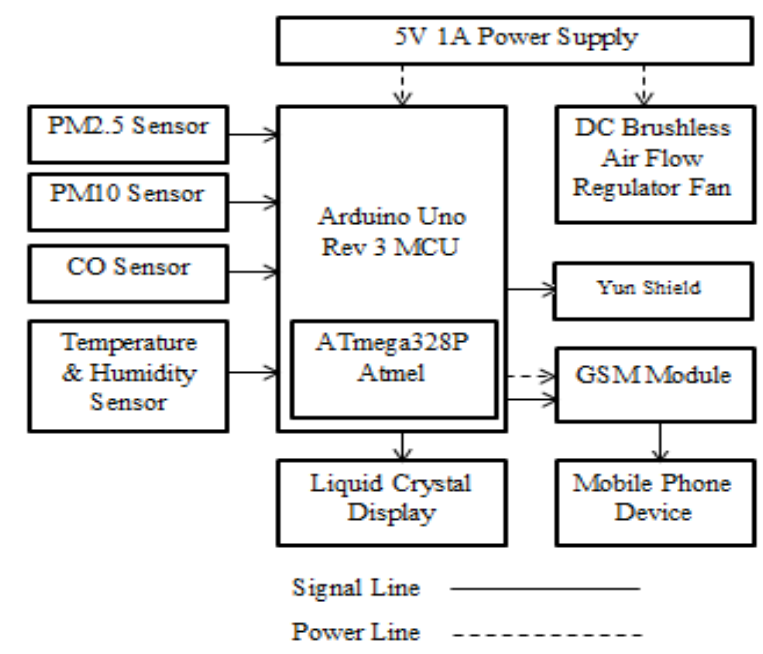

Figure 1. Detailed system block diagram of the API monitoring

\subsection{Sensor Selection}

The modules used in the system selected based on its functionality to carry out the task needed for the system. Microcontroller Arduino Uno Rev. 3 ATmega328P is enough to control the system. GSM Modem of SIM900 series used and with the dual network band, it can carry out its function to communicate with general mobile phones while LCD of 16x2 display used for nearby output visualization.

As in Table 2, the sensors integrated in the system are selected based on its measuring capability which is able to detect the pollutants concentration based on the Malaysia API scale. The higher the concentrations range of detection, the more expensive the sensor. Thus, wise selection of sensor in this project to make sure that the sensor is able to cover the API scale and still affordable in price. Figaro TGS 2600 sensor used to detect the $\mathrm{CO}$ concentrations in the surroundings area. It has capability of detecting several gaseous such as Methane, Iso-butane, ethanol, hydrogen and CO. It has high sensitivity towards low concentration of CO [16]. This sensor is metal-oxide semiconductor based sensor. The circuit built gives a signal which corresponds towards the change of pollutants that change the conductivity of sensor.

Table 2. Sensors detection range and API pollutants concentration range [2] [4] [14]

\begin{tabular}{llll}
\hline Sensors & Type of Pollutants & Detection Range & API Pollutants Concentration Range \\
\hline Figaro TGS 2600 & Carbon Monoxide & $1-100$ Part Per Million $(\mathrm{ppm})$ & $0-57.5 \mathrm{ppm}$ \\
SHARP DN7C3CA006 & PM2.5 & $25-500 \mu \mathrm{g} / \mathrm{m}^{3}$ & $0-500 \mu \mathrm{g} / \mathrm{m}^{3}$ \\
SHARP GP2Y1010AU0F & PM10 & $0-500 \mu \mathrm{g} / \mathrm{m}^{3}$ & $0-600 \mu \mathrm{g} / \mathrm{m}^{3}$ \\
DHT22 & Temperature \& & Humidity: 0-100\% & $\mathrm{n} / \mathrm{a}$ \\
& Humidity & Temperature: $-40-80^{\circ} \mathrm{C}$ & \\
\hline
\end{tabular}

The signal receives by the sensor is then computed by using functions obtained from the characteristics curve in the datasheet. The ratio between Sensor Resistance Ratio, $\mathrm{R}_{\mathrm{S}}$ and Sensor Resistance in Fresh Air, $\mathrm{R}_{\mathrm{O}}$ computed as follows;

$$
\alpha=\left\{\left[\left(\mathrm{V}_{\mathrm{CC}} \times \mathrm{R}_{\mathrm{L}}\right) / \mathrm{V}_{\text {OUTPUT }}\right]-\mathrm{R}_{\mathrm{L}}\right\} / \mathrm{R}_{\mathrm{O}}
$$

where $\alpha$ is the ratio between Sensor Resistance, $\mathrm{R}_{\mathrm{S}}$ and Sensor Resistance in Fresh Air, $\mathrm{R}_{\mathrm{O}}$ and $\mathrm{R}_{\mathrm{L}}$ is the Load Resistance [16].

DHT22 sensor has the capabilities to detect both temperature and humidity of the air. The computation of the sensor done in the sensor's library provided. No derivation of equation needed to obtain the results. This sensor uses the collective-digital-signal type method and humidity recognition and is calibrated readily [17]. For PM2.5 detection, SHARP DN7C3CA006 sensor will be selected. SHARP DN7C3CA006 is the third revision of PM sensor product by the SHARP Company. The sensor uses Optical dust sensing mechanism [18]. It has built-in fan for the air flow regulation through the sensing chamber. With its ability of measuring only dust with 2.5 micrometer of diameter and below, the severity in the haze environment can be known more 
detailed as PM2.5 has greater effect towards human health [20]. The results computed using the equation provided by the manufacturer in the datasheet.

SHARP GP2Y1010AU0F is a PM10 sensor and the first edition of the PM sensor from SHARP. The measurement of the sensor is based on the PM which has diameter of 10 micrometer and below. This version of sensor also using the optical sensing mechanism but doesn't have the air flow regulator. However, the air flow will be regulating by the installed DC brushless air flow regulator fan. An equation derived from the characteristic curve of the PM10 sensor to compute the results of detection as;

$$
\mathrm{PM} 10=(0.17 \times[\mathrm{VOUT} \times \mathrm{VCC} / 1024]-0.1)
$$

By having both PM2.5 and PM10, we can relatively know the composition of the dust based on its diameter. Hence, the severity of the dust contained in the air can be indicated clearly.

\subsection{Sensor Calibration}

Of all the sensors selected, the CO sensor, TGS 2600 is the only sensor that needs to be calibrated for consistent and reliable results. The sensor has been calibrated by referring a guided experimental research Error! Reference source not found.. $\mathrm{R}_{\mathrm{O}}$ is the constant which need to be recognized by calibration process. Using a closed space of $25 \mathrm{~m}^{3}, 24$ cigarettes smoked and $\mathrm{R}_{\mathrm{S}}$ measured after 3 hours as follows:

$$
\mathrm{R}_{\mathrm{S}}=\left[\left(\mathrm{V}_{\mathrm{CC}} \times \mathrm{R}_{\mathrm{L}}\right) / \mathrm{V}_{\text {OUTPUT }}\right]-\mathrm{R}_{\mathrm{L}}
$$

Based on the reference paper, $69.8 \mathrm{ppm}$ of $\mathrm{CO}$ resulted when 24 cigarettes smoked and contained for 3 hours without losses. By knowing the $\mathrm{CO}$ concentration, $\mathrm{R}_{\mathrm{O}}$ value obtained via equation (5) and (1) which is $28336.27 \Omega$.

Table 3. Percentage error between measured results and reference data Error! Reference source not found.

\begin{tabular}{cccc}
\hline No. Of Cigarettes & Reference CO PPM & Measured CO PPM & Percentage Error (\%) \\
\hline 4 & 12.21 & 13.06 & 6.96 \\
8 & 25.65 & 24.90 & 2.92 \\
16 & 47.63 & 46.10 & 3.21 \\
24 & 69.80 & 67.73 & 2.97 \\
\hline
\end{tabular}

To ensure the $\mathrm{R}_{\mathrm{O}}$ value obtained is acceptable, a number of cigarettes smoked by sequence from 4,8,16 and 24. For each session, the $\mathrm{CO}$ concentration measured after 3 hours of cigarettes smoked. The measured results compared with the reference data in Figure 2. The error calculated to analyze the deviation of measures results with reference data.

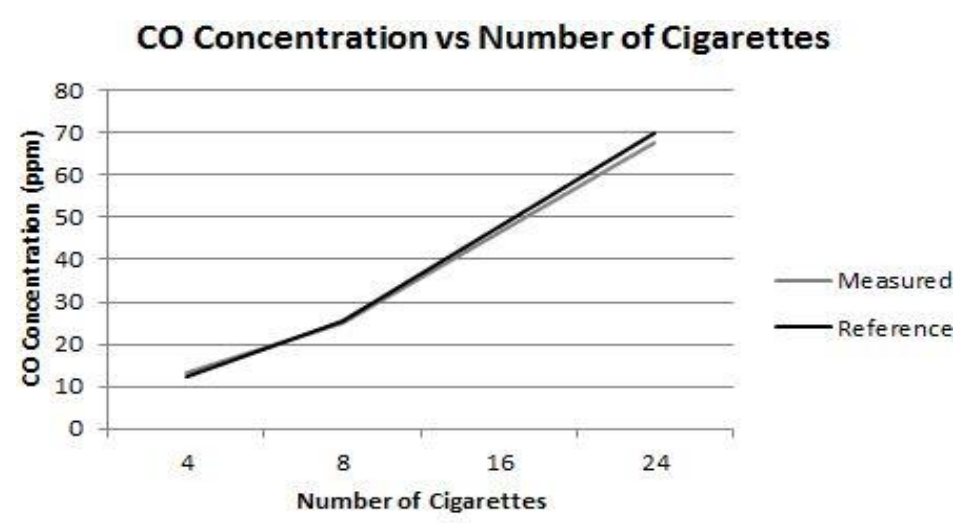

Figure 2. CO concentration vs. no. of cigarettes

Based on the comparison between the measured $\mathrm{CO}$ concentration and reference values, the deviation percentage is ranged between $2.92 \%-6.96 \%$. It is expected comes from the possible gas losses occurred during the calibration 


\subsection{Network Communication}

GSM SIM900A Modem used to establish a connection between Arduino Uno and Mobile Phone Device. AT Command used to communicate with the modem in order to gives order to perform intended actions i.e. sends and receives messages. General dual band used which enable the modem to connect with mobile phone devices of 900/1800MHz frequency bands. The API monitoring device will then send a short messaging service (SMS) to the user's mobile phone devices if the API reaches either Unhealthy, Very Unhealthy and Hazardous level.

\subsection{IoT Implementation on API Monitoring Device}

With the rise of the IoT trend, the possibilities for the API monitoring device to be IoT device have been explored. By transforming the API device to a remote sensing API device, it is connected to the Wi-Fi via Yun Shield Wi-Fi and Ethernet Module. ThingSpeak cloud web server used for the data storage of the sensors' data. ThingSpeak website projecting the data received by the device in the form of plotted graphs for each type of the pollutants. Hence, people will able to access the monitoring results and analyze the trends of the measured pollutants

\section{RESULTS AND ANALYSIS}

The integrated device tested to measure the surroundings air quality with real time monitoring. The device located at Universiti Teknologi PETRONAS, Seri Iskandar, Tronoh, Perak, Malaysia. Please refer to Figure 3.

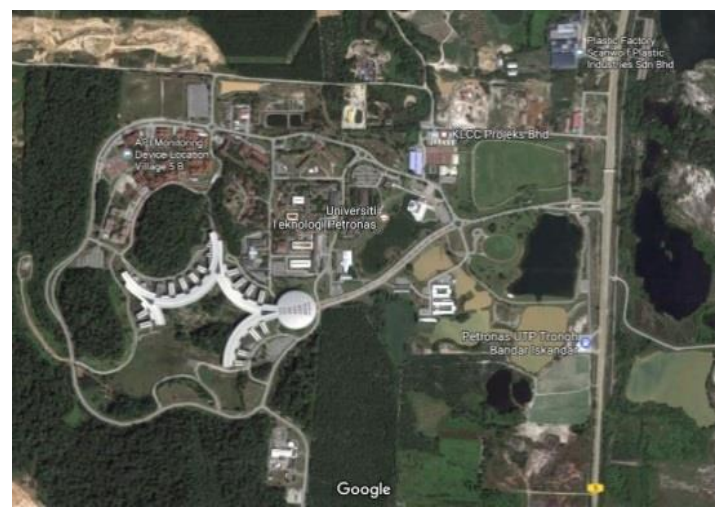

Figure 3. API monitoring device location

\subsection{CO Concentration Monitoring}

Figure 4 shows an example of 1 day of CO concentration monitoring through the ThingSpeak website. The overall results show decrement from 0100 hour until 0600 hour and start to increase until the peak hour. This trend suspected due to the peak working hours. The peak hours for $\mathrm{CO}$ concentration measured highest at 1000 hour. The result from 1100 to 1500 hour shows significant decrement. One of the possible reason is the $\mathrm{CO}$ contain in the air reduced as the human working activities decreasing. There was a significant increase from 1500 until 1800 and fluctuations observed from 1800 until 0000 hour. The discrepancies of CO concentration levels observed influenced by the weather conditions, human activities, wind directions and its sources.

This 1-day monitoring result obtained from ThingSpeak website have not much difference of trend to the 5 weeks monitoring results. It is shown that for all of figures, there are similarities of trends which indicates the stabilities and consistency of sensor performance. Based on the Figure 5, the data trend shown quite similar to the 5-week monitoring. 


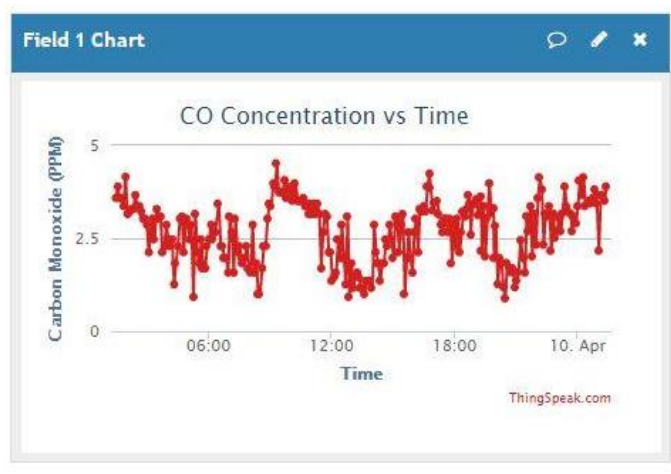

Figure 4. Example of co monitoring data from thingspeak website

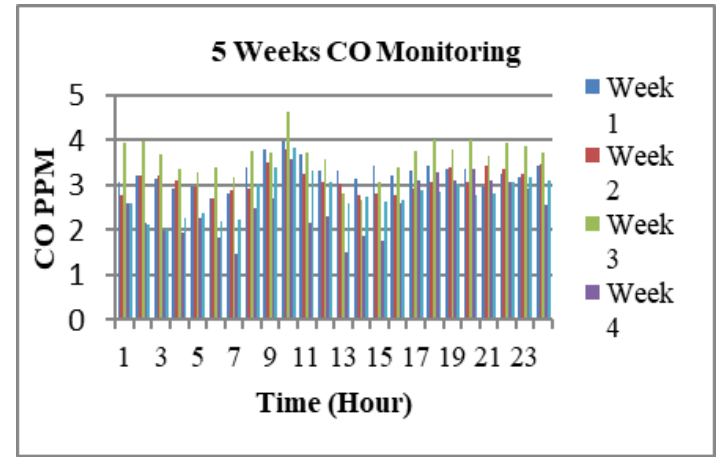

Figure 5. Five weeks CO monitoring

\subsection{Temperature Monitoring}

Figure 6 shows the single day monitoring result obtained from ThingSpeak for temperature monitoring. Generally, from 0100 hour, a decrement observed until 0800 hour. The temperature starts to increase from 0900 hour until its peak hour where the highest temperature recorded everyday which is at 1600 hour. This highest temperature recorded at 1600 hour because direct sunlight towards the API monitoring device happens at that hour. Subsequently, decrement observed from 1700 until 0000 hour.

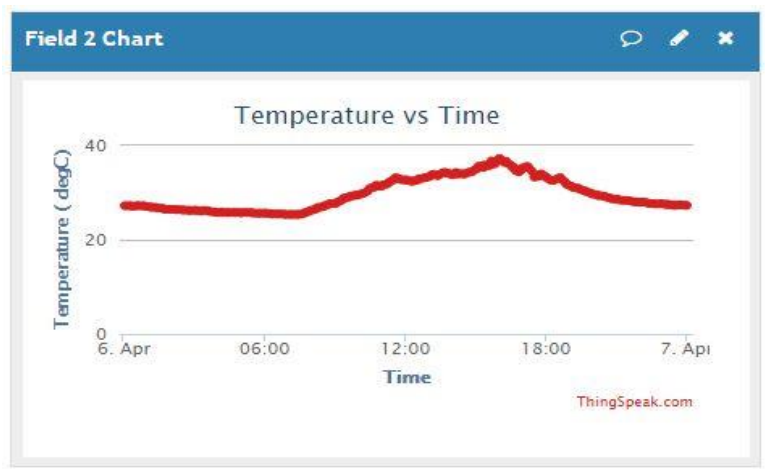

Figure 6. Example of temperature result from thingspeak website

In Figure 7, 5 weeks of temperature monitoring have been monitored. Based on the result, the highest peak hours of temperature monitored around 1600-hour. The results plotted has same trend as the 1 days of temperature monitoring.

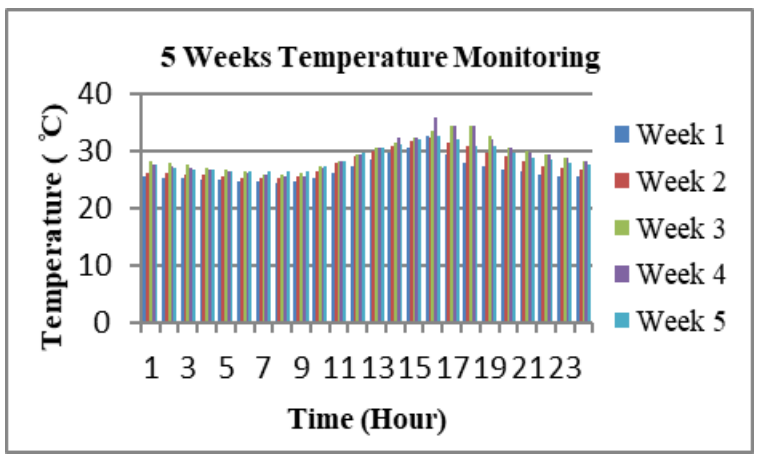

Figure 7. Five weeks temperature monitoring 


\subsection{Humidity Monitoring}

Figure 8 shows the 1 of humidity monitoring from ThingSpeak Website. 7 From 0100 hour until 0800 hour, increment on humidity observed and the peak hour recorded at 0800 . There is significant decrement from 0900 hour to 1600 hour and the lowest humidity recorded is at 1600 every day. Subsequently, the humidity changes with increment from 1700 until 0000 hour.

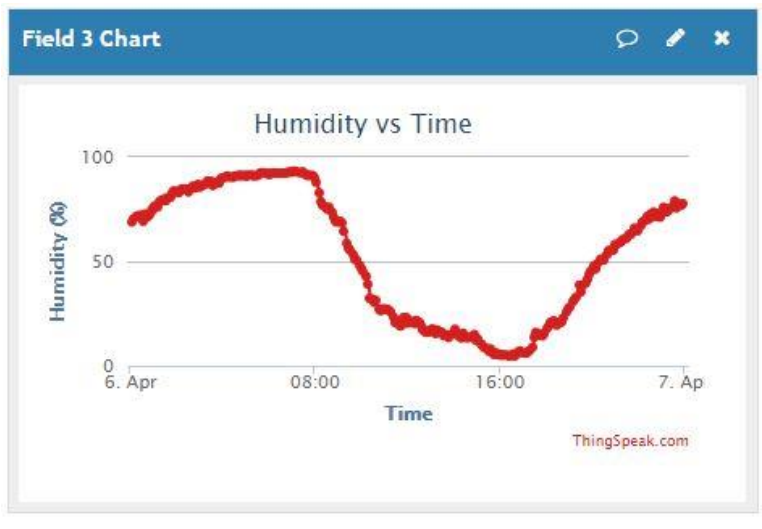

Figure 8. Example of humidity monitoring data from thingspeak website

Based on the Figure 9, the lowest peak hours of humidity monitored around 1600-hour. The results plotted has same trend as the 1 days of humidity monitoring from ThingSpeak Website.

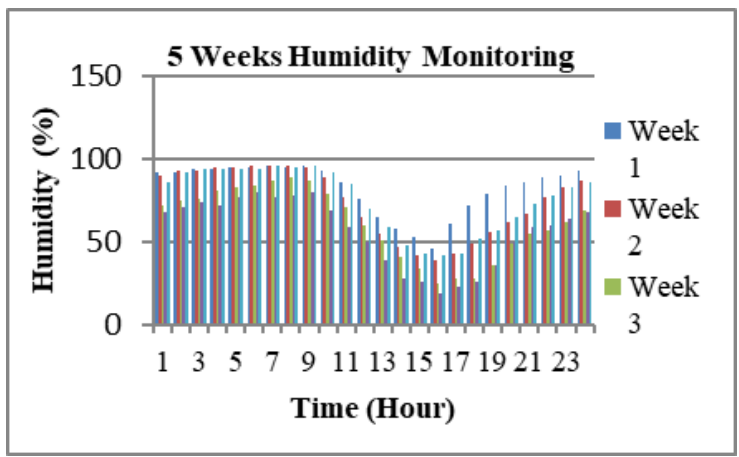

Figure 9. Five weeks humidity monitoring

Generally, the humidity results are somehow correlated with the temperature results obtained. The humidity and temperature have inverse relationship with each other. This clearly can be seen where the peak hour of humidity corresponds to the lowest temperature recorded which is at 0800 hour. On the other hand, the lowest humidity recorded corresponds to the peak hour of temperature which at 1600 hour. Similar to the temperature results, humidity results are affected by the changes of weather.

\subsection{PM2.5 and PM10 Monitoring}

Based on Figure 10, the PM10 result shows that there is no PM value being measured because of the location factor where the monitoring location does not have many industrial activities. Furthermore, the sensor also has less sensitivity and required a significant change of environment to ensure its detection. As a result, a PM2.5 was introduced as part of sensors the air monitoring quality system. 


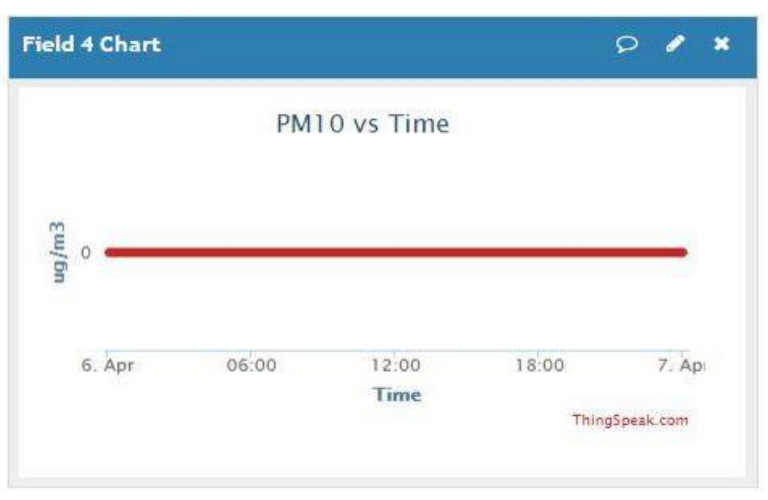

Figure 10. Example of PM10 monitoring data from thingspeak website

In Figure 11, the trend of the PM value was now able to be monitored. It was observed that the peak hours for PM2.5 measured highest from 1000 hour to 13 hour.

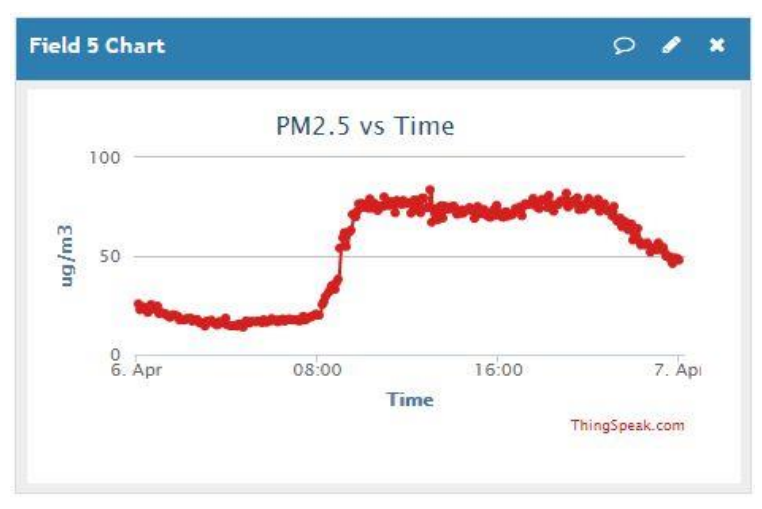

Figure 11. Example of PM2.5 monitoring data from thingspeak website

The trends of the 5 days PM2.5 have been exported into excel and analyzed by the mean values as in Figure 12. The sensitivity of PM2.5 can detect the particle changes with better sensitivity compared to PM10. This shows that PM2.5 is important component and need to be added as part of the air quality monitoring system.

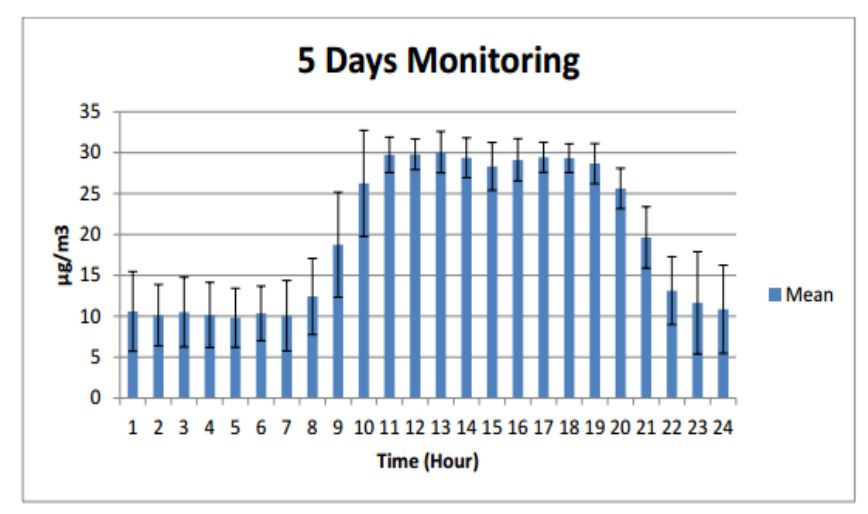

Figure 12. Five days of PM2.5 monitoring data 


\section{CONCLUSION}

In brief, the focus of his project is to develop an affordable and dependable IOT prototype for real time air quality monitoring. The necessity of knowing the level or condition of the quality of the air we breathe in our surroundings is very crucial in order to keep our good health condition. The proposed system will be able to solve Malaysians concern on the delay announcement and measuring the PM2.5 haze in Malaysia considering the Malaysia new API system will be only available in 2020. The PM2.5 measurements is very essential to give clearer dust pollutants condition to ensure healthy life environment. The prototype developed was able to successfully monitor the Air Pollution Index at UTP for 5 weeks.

\section{ACKNOWLEDGEMENTS}

The authors would like to acknowledge Universiti Teknologi PETRONAS(UTP), Malaysia for sponsoring this research work.

\section{REFERENCES}

[1] Mabahwi N. A., Leh O. L. H. and Omar D., "Urban Air Quality and Human Health Effects in Selangor," Malaysia. Procedia - Social and Behavioral Science, vol. 170(1), pp. 282-291, 2015.

[2] Last Minute Notice to Close Schools Irks Malaysians. Retrieved on September 30 $0^{\text {th }}$, 2016, from http://www.thestar.com.my/news/nation/2015/09/16/lastminute-notice-to-close-schools-irks-malaysians

[3] Department of Environment Malaysia, "A Guide to Air Pollutant Index (API) in Malaysia $4^{\text {th }}$ Edition," Malaysia: ASMA Sdn. Bhd, 2000.

[4] Air Pollution Index of Malaysia. Retrieved on September $24^{\text {th }}, 2016$, from http://apims.doe.gov.my/v2/

[5] Singapore National Environment Agency. Retrieved on January 31 $1^{\text {st }}, 2017$, from http://www.haze.gov.sg/

[6] Liu J. H. et al., "An Air Quality Monitoring System for Urban Areas Based on the Technology of Wireless Sensor Network," International Journal on Smart Sensing and Intelligent Systems, vol. 5(1), pp. 191-214, 2012.

[7] N. Kularatna and B. H. Sudantha, "An Environmental Air Pollution Monitoring System Based on the IEEE 1451 Standard for Low Cost Requirement," IEEE Sensor Journal, vol. 8(4), pp. 415-422, 2008.

[8] AEA Technology Plc, "A Guide for Local Authorities Purchasing Air Quality Monitoring Equipment $2^{\text {nd }}$ Edition," United Kingdom: AEA Technology Plc, 2006.

[9] Ruslan N., "Air Pollution Index Real Time Monitoring System," Malaysia: Universiti Tun Hussein Onn, 2015.

[10] Shariff S. S., "Real Time Air Quality Reporting System," Malaysia: Universiti Tun Hussein Onn, 2015.

[11] Suyuti A. et al., "Microcontroller ATMEGA8535 based design of Carbon Monoxide Gas Detector," International Journals of Engineering and Science, vol. 12(4), pp. 71-80, 2012.

[12] Edward O. O., "A Microcontroller Based Data Acquisition System for Industrial Air Pollution Concentration Measurement in Nigeria," Journal of Multidisciplinary Engineering Science and Technology, vol. 1(3), pp. 19-24. 2014.

[13] Witte T. F.,"Development of An Indoor Air Quality Monitoring System based on a Microcontroller," Finland: Helsinki Metropolia University of Applied Sciences, 2014.

[14] J. Dutta, F. Gazi, S. Roy and C. Chowdhury, "AirSense: Opportunistic Crowd-Sensing Based Air Quality Monitoring System for Smart City," 2016 IEEE SENSORS, Orlando, FL, pp. 1-3, 2016.

[15] G. B. Fioccola, R. Sommese, I. Tufano, R. Canonico and G. Ventre, "Polluino: An Efficient Cloud-Based Management of Iot Devices for Air Quality Monitoring,"2016 IEEE 2nd International Forum on Research and Technologies for Society and Industry Leveraging a better tomorrow (RTSI), Bologna, pp.1-6, 2016.

[16] Revised AQI Breakpoints. Retrieved on December $4^{\text {th }}$, 2016, from http://aqicn.org/faq/2013-09-09/revised-pm25aqi-breakpoints/

[17] FIGARO USA INC, "TGS 2600 for Detection of Air Contaminants," TGS 2600 Datasheet, Jan 2005. [Revised on Dec 2016].

[18] Adafruit Industries LLC, "Digital Relative Humidity \& Temperature Sensor AM2302/DHT22," [Revised on Feb 2017].

[19] SHARP Corporation, "PM2.5 Sensor Module," DN7C3CA006 Datasheet, September 2014. [Revised on Dec 2016].

[20] SHARP Corporation, "Compact Optical Dust Sensor," GP2Y1010AUOF Datasheet, Dec 2006. [Revised on Dec 2016].

[21] Xing Y.F, Xu Y.-H., Shi M.-H., and Lian Y.-X.,"The Impact of Pm2.5 On the Human Respiratory," system Journal of Thoracic Disease, vol. 8(1), pp. E69-E74, 2016.

[22] Hoegg U. R., "Cigarette Smoke in Closed Spaces," Environmental Health Perspectives, vol. 2, pp. 117128, 1972. 